\title{
Design and Optimization of a Monoaxial Tracking System for Photovoltaic Modules
}

\author{
Cătălin Alexandru \\ Transilvania University of Braşov, 29 Eroilor Boulevard, 500036 Braşov, Romania \\ Correspondence should be addressed to Cătălin Alexandru; calex@unitbv.ro
}

Received 28 January 2013; Revised 12 June 2013; Accepted 2 July 2013

Academic Editor: Koray Ulgen

Copyright (C) 2013 Cătălin Alexandru. This is an open access article distributed under the Creative Commons Attribution License, which permits unrestricted use, distribution, and reproduction in any medium, provided the original work is properly cited.

This paper presents researches on increasing the energetic efficiency of a photovoltaic (PV) string by designing and optimizing a tracking mechanism that simultaneously changes the daily position of the modules using a single driving source (there are modules with individual supports). The motion is transmitted from the driving source, which is a linear actuator, with a parallelogram mechanism. The main task in optimizing the tracking system is to maximize the energetic gain by increasing the solar input and minimizing the energy demand for tracking. The study is performed by developing the virtual prototype of the tracking system, which integrates the mechanical device and the control system, in mechatronic concept. Virtual prototyping software solutions (ADAMS, EASY5, and MAT) are used in this study.

\section{Introduction}

The realization of the PV strings (system of PV modules that function as a single electricity-producing unit) appeared as a necessity for the development of large systems for producing electricity. The energetic efficiency of the PV strings depends on the degree of use of the solar radiation [1], which can be maximized by use of tracking systems. These are mechatronic devices, which ensure the optimal positioning of the string relative to the Sun's position.

Depending on the degree of mobility, there are two basic types of tracking systems: monoaxis and dual-axis systems. The monoaxis trackers perform only the daily motion, the tilt angle of the motion axis corresponding to the latitude angle of the location [2], while the dual-axis trackers perform both motions (daily and seasonal/elevation), so that they are able to follow very precisely the Sun path throughout the year. The dual-axis tracking systems can increase the energetic efficiency up to $40-45 \%$ against the equivalent fixed systems, while the energy gain for the mono-axis systems is lower (up to $30-35 \%$ ) [3-6].

From energetic point of view, the PV string with tracking is efficient if the energy that it produces $\left(E_{T}\right)$ is substantially greater than the sum of the energy produced by the equivalent string without tracking/fixed $\left(E_{F}\right)$ and the energy demand for orientation $\left(E_{C}\right)$,

$$
E_{T} \gg E_{F}+E_{C} .
$$

The optimal design of the tracking system, aiming to maximize the energetic efficiency, has become an important challenge in the modern research and technology.

In practice, the orientation of the PV strings can be realized in two ways: independent orientation for each module of the string (module with its own tracking system, motor source); simultaneous orientation of the modules from the same motor source, with the help of motion transmitting mechanisms. The simultaneous orientation, with the predicted advantages (coming mainly from minimizing the number of motor sources) and the characteristic problems, opens a research area insufficiently explored since now.

In the literature, there is no unitary modeling for the tracking mechanisms of the PV strings referring to the structural, kinematical, and dynamical issues. At the same time, there is no general approach for conceptual design and structural synthesis of these mechanisms. Thereby the necessity of a method for the unitary modeling of the tracking mechanisms become obvious, and according to the strategy proposed by the paper this method is based on the MBS 
(Multibody Systems) theory [7, 8], which facilitates the selfformulating algorithms.

There are several works that approach control issues, mostly for the tracking systems of the individual modules, using different techniques (closed loop systems with photo sensors, open loop systems based on astronomical computerized systems, or hybrid combinations), and controllers (PID, FNC, and FNLC) [9-12]. The research is focused mostly on the evaluation/prediction of the energy achieved by tracking and less on the energy demand for performing the orientation.

There are different models for the evaluation of the radiation potential as input data in the tracking systems design. The solar radiation can be measured using traditional instruments or can be digitally recorded with a data acquisition system. At the same time, there were developed large meteorological databases, such as Meteonorm. The traditional Angstrom's linear approach is based on measurements of sunshine duration, while relatively new methods are based on artificial neural networks-ANN [13]. Several models for estimating the monthly mean solar radiation (linear Angstrom-Prescott variation, quadratic equation, logarithmic variation, and exponential function) were studied in [14], the root mean square error being the main element of the comparative analysis. The mathematical model developed in [15] is used for estimating the hourly and daily radiation incident on three-step tracking planes.

In these terms, the present paper approaches the improvement of the energetic efficiency of a PV string by designing and optimizing a mono-axis Sun tracker that simultaneously changes the daily position of the PV modules with a single driving source. The main task in optimizing the mechanism is to maximize the energetic gain by increasing the solar radiation input and minimizing the energy demand for tracking. The incident radiation is estimated by using a mathematic algorithm based on the direct terrestrial radiation and the angle of incidence. The paper proposes the integration of the mechanical and electronic (control) components at the virtual prototype level (i.e., modeling in mechatronic concept), which allows performing the energy balance of the PV system.

\section{Designing the Tracking System}

The system approached in this paper corresponds to a monoaxis tracking mechanism (Figure 1), at which the daily motion is transmitted from the motor source, which is a linear actuator, with a multiparallelogram mechanism, the revolute axes of the PV modules (in this case, three modules) being parallel with the polar axis. The design is made for an inline string configuration, but it can be adapted for other configurations or for a higher number of modules. The driving actuator is disposed on a fixed frame, and this has positive effect on the mass and inertia loading in the tracking mechanism.

The structural model of the tracking system is shown in Figure 2, the representation being made in the motion plan $(\Pi)$, which is normal to the polar axis. The geometric

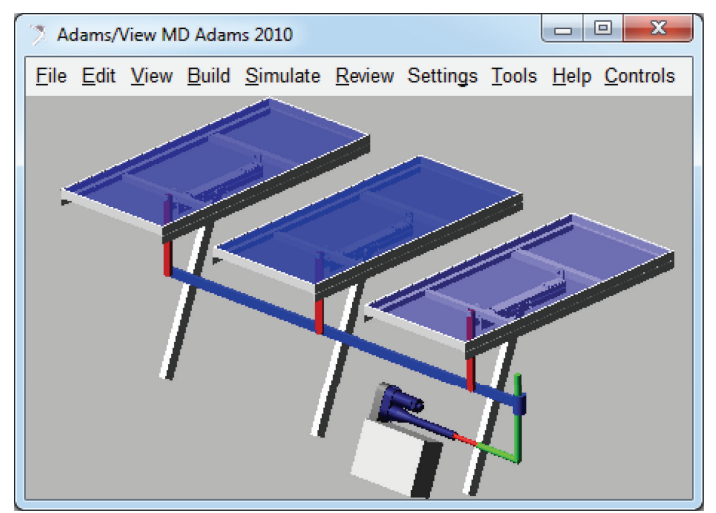

FIGURE 1: The virtual prototype of the PV string with monoaxis tracking system.

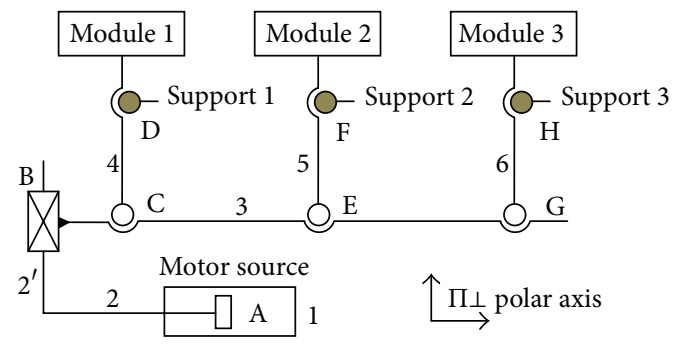

Figure 2: The structural model of the tracking mechanism.

constraints from $\mathrm{A}$ and $\mathrm{B}$ are translational joints, while the others $(\mathrm{C}, \ldots, \mathrm{H})$ are revolute joints. The intermediary element $2^{\prime}$, which moves rod 3 of the parallelogram mechanism, is rigidly connected to the actuator piston 2. Each module rotates around its own support, which is fixed on the ground.

For simulating the dynamic behavior of the tracking system, the virtual prototype was developed in mechatronic concept by using a digital software platform, which integrates the following components: CAD—Computer Aided Design (CATIA) - to create the solid model, MBS-MultiBody Systems (ADAMS) - for analyzing and optimizing the mechanical device, and DFC-Design for Control (EASY5 and ADAMS/Controls) - for the control system design.

For connecting the mechanical model and the electronic control system, the input and output plants have been defined. The control force generated by the linear actuator represents the input parameter in the mechanical model. The output transmitted to the controller is the position angle of the modules (i.e., the daily angle). The input and output plants are saved in a specific file for EASY $5\left({ }^{*}\right.$.inf), which is used to create the control system diagram (Figure 3 ). In the control system model, the TI block (tabular function of time) is the database of the imposed daily angle, while the MSC.ADAMS block includes the mechanical device plant.

From the controller point of view, for obtaining reduced transitory period and small errors, a generic control loop feedback mechanism (PID controller) was used. The controller tuning (aiming to obtain the proportional P, Integral I, and derivative $\mathrm{D}$ terms) was performed in an optimal design process with Matrix Algebra Tool (MAT), the model being 


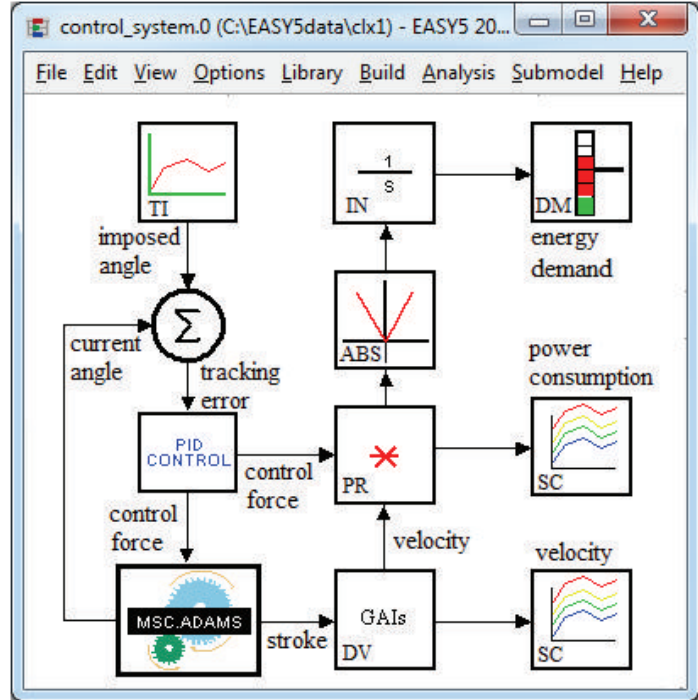

FIgURE 3: The control system block diagram.

transferred to MAT by using the EMX file format. The design objective refers to the minimization of the tracking error (the difference between the imposed and current daily angle; see Figure 3).

In MAT, the optimization was performed by using the "minimize_v" function:

$$
[x, f]=\text { minimize } \_v\left(\text { function_name, } x_{0}, H_{0} \text {, tol, delx }\right) \text {, }
$$

where $x_{0}$ is the initial guess for minimizer, $H_{0}$ the initial guess for Hessian, tol the relative tolerance for $x$, delx the relative step size for computing gradients by differencing, $\mathrm{x}$ the minimizer of the function, and $f$ the value of the function at $x$.

The next step was to create the MAT minimizer function that performs the optimization. The study is performed by calling the "minimize $v$ " function with the minimizer function as the first argument; MAT will repeatedly call the function as it performs the minimization procedure. The function will set the tracking error appropriately and return the error in the simulation, defined as the sum of the squares of the differences between the simulation and desired values. The values of the proportional, integral, and derivative terms will result in a simulation that meets the design requirements as follows: $\mathrm{P}=2150, \mathrm{I}=1503$, and $\mathrm{D}=1405$.

Finally, in the cosimulation process, ADAMS accepts the control force from EASY5 and integrates the mechanical model in response to this. At the same time, ADAMS provides the current daily angle for EASY5 to integrate the control system model.

\section{Designing the Tracking Law}

The PV modules can be rotated without brakes during the daylight or can be discontinuously driven (step-by-step motion). The key idea for optimizing the motion law is to maximize the energy gained through the step-by-step orientation, for absorbing a quantity of solar energy closed by the ideal case (continuous orientation), and to minimize the energy demand for performing the tracking.

The energy produced by the PV string depends on the quantity of incident radiation, the module efficiency, and the number of modules. The incident radiation depends on the direct terrestrial radiation and the angle of incidence. The direct radiation is established using the Meliß's empirical model [16], depending on the extraterrestrial radiation, the medium solar constant, the day number during a year, the atmosphere clarity, the solar altitude angle, the solar declination, the latitude angle, the solar hour angle, and the solar time.

The incidence angle is determined from the scalar product of the sunray vector and the normal vector on module, depending on the diurnal and seasonal angles of the sunrays, the daily and elevation angles of the module, and the azimuthal angle [3]. In this way, the incident solar radiation can be estimated in every day during a year, for different locations and tracking strategies.

The paper presents the exemplification for the summer solstice day, the numeric simulations being performed for the Braşov geographic area, with the following input data: the location latitude, $\varphi=45.5^{\circ}$; the solar declination, $\delta=23.45^{\circ}$; the day number during the year, $n=172$ (June 21); the local time (from sunrise to sunset), $T \in(5: 35,21: 04)$.

For identifying the optimal motion field, there was considered the correlation between the motion amplitude and the local time, for obtaining symmetric revolute motions relative to the solar noon position $\left(\beta^{*}=0, T=13: 19\right)$. The analysis has been performed for the following cases: (a) $\beta^{*} \in\left[+90^{\circ},-90^{\circ}\right], T \in[5: 35,21: 04]$, the maximum motion field; (b) $\beta^{*} \in\left[+75^{\circ},-75^{\circ}\right], T \in[6: 52,19: 46]$; (c) $\beta^{*} \in\left[+60^{\circ},-60^{\circ}\right], T \in[8: 10,18: 29] ;$ (d) $\beta^{*} \in\left[+45^{\circ},-45^{\circ}\right]$, $T \in[9: 27,17: 11] ;(\mathrm{e}) \beta^{*} \in\left[+30^{\circ},-30^{\circ}\right], T \in[10: 44,15: 53]$; (f) $\beta^{*} \in\left[+15^{\circ},-15^{\circ}\right], T \in[12: 02,14: 36]$. In addition, for the fixed (nontracked) string, $\beta^{*}=0$ throughout the daylight $(T \in[5: 35,21: 04])$. In this study, the PV modules are rotated without brakes (continuous motion). After sunset (21:04), the tracking system returns to the initial position for the next day (facing East), on the same route, with continuous motion.

In this way, the incident radiation curves have been obtained (Figure 4). Integrating these curves and taking into account the number of modules in the string ( 3 modules), the active surface of each module $\left(1.26 \mathrm{~m}^{2}\right)$, and the PV module efficiency (i.e., the solar radiation conversion rate) $(15 \%)$, the energy produced by the PV string (with and without tracking) has been obtained.

Afterwards, the energy demand for realizing the motion laws was determined by using the virtual prototype of the tracking system. The return of the tracking system in the initial position is also considered. In this way, the energy balance was performed, the results being systematized in Table 1 . The energy gain $(\varepsilon)$ is computed relative to the fixed string:

$$
\varepsilon=\left[E_{T}-\left(E_{F}+E_{C}\right)\right] \cdot \frac{100}{E_{F}} .
$$




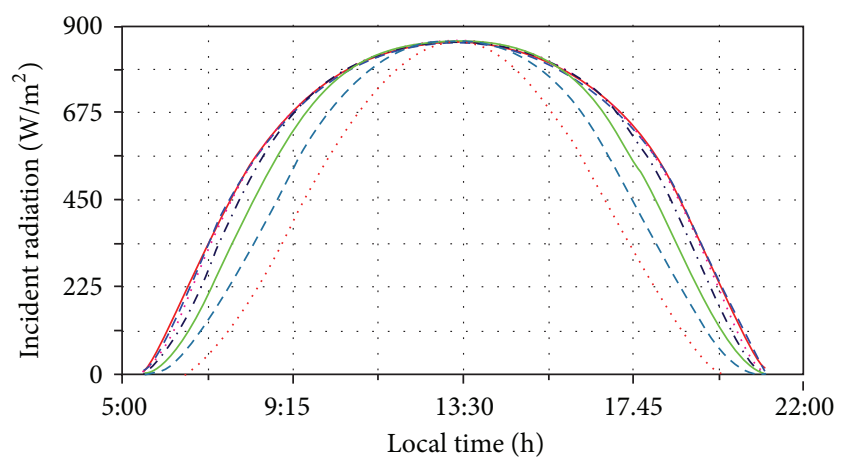

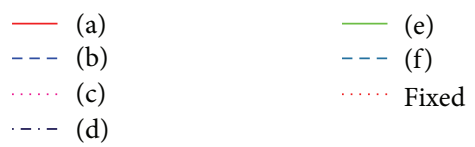

FIGURE 4: The incident radiation curves for the continuous tracking cases.

TABLE 1: The energy balance for the continuous tracking cases.

\begin{tabular}{lcccc}
\hline $\begin{array}{l}\text { Tracking } \\
\text { case }\end{array}$ & $E_{T}[$ Wh/day $]$ & $E_{F}[$ Wh/day $]$ & $E_{C}[$ Wh/day $]$ & $\varepsilon[\%]$ \\
\hline (a) & 5222.63 & & 52.90 & 40.02 \\
(b) & 5218.21 & & 39.97 & 40.25 \\
(c) & 5179.33 & 3692.18 & 24.35 & 39.62 \\
(d) & 5056.86 & & 15.32 & 36.55 \\
(e) & 4796.72 & & 7.01 & 29.73 \\
(f) & 4348.82 & & 1.78 & 17.74 \\
\hline
\end{tabular}

Because the energy intake brought by the tracking cases (a) and (b) is very small comparative with (c), the case (c) has been chosen as optimal; in other words the optimal field for the daily angle of the PV string is $\beta^{*} \in\left[+60^{\circ},-60^{\circ}\right]$. In the same idea, as can be seen in Figure 4, the solar radiation has small values in the limit positions, close to the sunrise and sunset, and for this reason it is not efficient to track the Sun in these areas.

The continuous orientation (without brakes) was used only for establishing the optimal angular field of the daily motion. In the next stage, the step-by-step tracking is implemented to avoid the continuous orientation disadvantages, such as: the high operating time of the system, which has negative influence on the reliability; the need to achieve large transmission ratios, which can cause constructive issues; the behavior of the system under the action of external perturbations (e.g., wind), whose effect can be amplified if the system is moving.

Under these circumstances, several step-by-step tracking strategies have been evaluated, depending on the number of steps (in consequence, the step dimension $\Delta \beta^{*}$ ) for realizing the optimal angular field of the daily motion $\beta^{*} \epsilon$ $\left[+60^{\circ},-60^{\circ}\right]: 12$ steps $\left(\Delta \beta^{*}=10^{\circ}\right), 10$ steps $\left(\Delta \beta^{*}=12^{\circ}\right), 8$ steps $\left(\Delta \beta^{*}=15^{\circ}\right), 6$ steps $\left(\Delta \beta^{*}=20^{\circ}\right), 4$ steps $\left(\Delta \beta^{*}=30^{\circ}\right)$, or 2 steps $\left(\Delta \beta^{*}=60^{\circ}\right)$.

One of the most important problems in the step-bystep tracking is to identify the optimal actuating time.

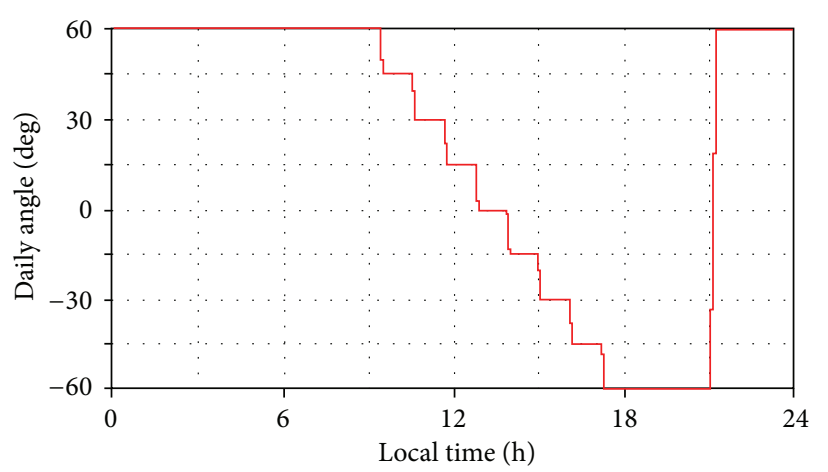

FIGURE 5: The 8-step tracking law.

TABLE 2: The energy balance for the step-by-step tracking cases.

\begin{tabular}{|c|c|c|c|c|c|}
\hline $\begin{array}{l}\text { Number of } \\
\text { steps }\end{array}$ & $E_{T}[\mathrm{Wh} /$ day $]$ & $E_{F}[\mathrm{Wh} /$ day $]$ & $E_{C}[\mathrm{Wh} /$ day $]$ & $\varepsilon[\%]$ & $\varepsilon_{t}[\%]$ \\
\hline 12 & 5161.40 & \multirow{6}{*}{3692.18} & 28.68 & 39.02 & 298.48 \\
\hline 10 & 5159.17 & & 27.588 & 38.99 & 98.40 \\
\hline 8 & 5155.06 & & 26.874 & 38.89 & 98.17 \\
\hline 6 & 5146.18 & & 26.214 & 38.67 & 797.60 \\
\hline 4 & 5120.75 & & 25.158 & 38.01 & l 95.94 \\
\hline 2 & 5012.90 & & 24.36 & 35.11 & 88.62 \\
\hline
\end{tabular}

The solution is obtained by developing an algorithm based on the following phases: the optimal angular field is segmented into the intermediary positions depending on the step dimension for each case (e.g., for 8 steps there are the following positions: $\beta^{*}=\left\{ \pm 60^{\circ}, \pm 45^{\circ}, \pm 30^{\circ}, \pm 15^{\circ}, 0^{\circ}\right\}$ ), and the incident radiation curves are consecutively obtained considering the module fixed in these positions during the daylight; analyzing these curves, the moment in which the value of the incident radiation for a certain position " $k$ " becomes smaller than the value in the next position " $k+1$ " is identified; the analysis continues with the next pair of positions " $k+1$ " and " $k+2$ " and so on. For example, Figure 5 shows the 8-step tracking law which has been obtained in accordance with the previous described algorithm.

For the considered step-by-step tracking cases, the results of the energy balance are systematized in Table 2. The energy gain $(\varepsilon)$ is computed relative to the fixed string case (see (3)), while the step-by-step tracking efficiency $\left(\varepsilon_{t}\right)$ is determined as relative value to the energy gain of the optimal continuous tracking case, with the corresponding value from Table 1, case "c" $\left(\varepsilon^{(c)}=39.62 \mathrm{Wh} /\right.$ day $)$, being as follows:

$$
\varepsilon_{t}=\varepsilon \cdot \frac{100}{\varepsilon^{(c)}}
$$

The energy demand for realizing the step-by-step motion laws is a little bit greater than the energy demand for the continuous motion, and this is because of the overshootings that appear when the actuator is started (each step meaning an actuator starter). However, the differences in energy demand are relatively small, there being the same linear travel of the actuator (i.e., the same angular travel of the PV 


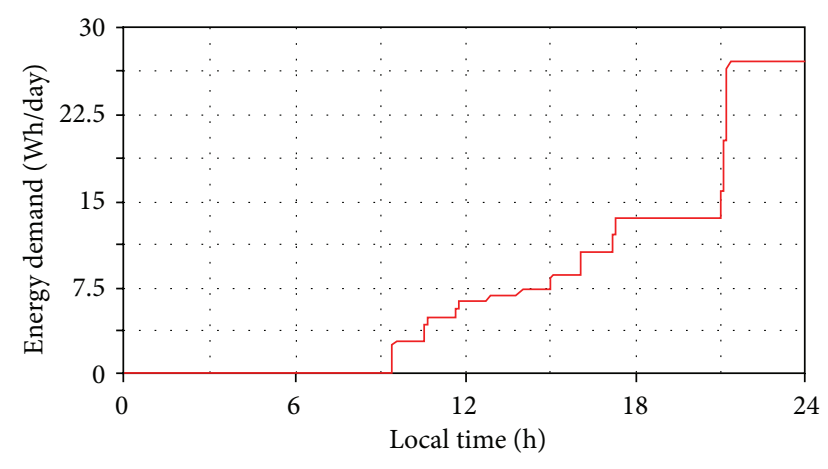

Figure 6: The energy demand for the 8-step tracking law.

modules). For instance, the energy demand for the 8-step tracking case is shown in Figure 6.

Therefore, by using the proposed algorithm for configuring the step-by-step tracking, obtained values (in terms of energy gain) are close to those of the continuous tracking (which is not viable for physical implementation due to the aforementioned disadvantages), and this demonstrates the viability of the adopted optimization strategy. Concerning the optimal number of tracking steps, it was demonstrated that the amount of energy gain per step decreases when the order number of the step increases $[17,18]$. The energy brought by the final step has to be greater than the energy demand for realizing this step.

\section{Conclusions}

The application is a relevant example regarding the implementation of the virtual prototyping tools in the design process of the tracking systems. One of the most important advantages of this kind of simulation is the possibility to perform virtual measurements in any area and for any parameter (motion, force, energy). At the same time, by integrating the electronic control system and the mechanical device of the tracking mechanism at the virtual prototype level, the physical testing process is greatly simplified, and the risk of the control law being poorly matched to the real photovoltaic tracking system is minimized.

The optimization strategy based on the minimization of the angular field for the daily motion and the determination of the optimal actuating time to perform the motion steps leads to an efficient PV system, without developing expensive hardware prototypes. In this way, the behavioral performance predictions are obtained much earlier in the design cycle, thereby allowing more effective and cost efficient design changes.

Considering the algorithm of the product design development, the virtual prototype precedes the manufacturing and implementation stage. Based on the simulation and optimization results (some of them being presented in this paper), the embodiment design has been recently finished, and the technical documentation for manufacturing has been elaborated. The physical prototype of the tracking system is about to be developed, and it will be implemented in the Green Energy Independent University Campus. This will allow a relevant comparison between the virtual prototype analysis and the data achieved by measurements.

\section{References}

[1] G. N. Tiwari, Solar Energy, Alpha Science Int. Ltd., Pangbourne, UK, 2002.

[2] E. Calabro, "An algorithm to determine the optimum tilt angle of a solar panel from global horizontal solar radiation," Journal of Renewable Energy, vol. 2013, Article ID 307547, 12 pages, 2013.

[3] C. Alexandru and C. Pozna, "Simulation of a dual-axis solar tracker for improving the performance of a photovoltaic panel," Proceedings of the Institution of Mechanical Engineers A, vol. 224, no. 6, pp. 797-811, 2010.

[4] C. Alexandru and I. N. Tatu, "Optimal design of the solar tracker used for a photovoltaic string," Journal of Renewable and Sustainable Energy, vol. 5, no. 2, Article ID 023133, pp. 1-16, 2013.

[5] S. Seme and G. Štumberger, "A novel prediction algorithm for solar angles using solar radiation and Differential Evolution for dual-axis sun tracking purposes," Solar Energy, vol. 85, no. 11, pp. 2757-2770, 2011

[6] G. K. Singh, "Solar power generation by PV, (photovoltaic) technology: a review," Energy, vol. 53, pp. 1-13, 2013.

[7] M. Ceccarelli, "Challenges for mechanism design," in Proceedings of the 10th IFToMM International Symposium on Science of Mechanisms and Machines, pp. 1-13, 2009.

[8] W. Schiehlen, "Multibody systems: roots and perspectives," Multibody System Dynamics, vol. 1, no. 2, pp. 149-188, 1997.

[9] K. S. Karimov, M. A. Saqib, P. Akhter, M. M. Ahmed, J. A. Chattha, and S. A. Yousafzai, "A simple photo-voltaic tracking system," Solar Energy Materials and Solar Cells, vol. 87, no. 1-4, pp. 49-59, 2005.

[10] F. R. Rubio, M. G. Ortega, F. Gordillo, and M. López-Martínez, "Application of new control strategy for sun tracking," Energy Conversion and Management, vol. 48, no. 7, pp. 2174-2184, 2007.

[11] S. Abdallah and S. Nijmeh, "Two axes sun tracking system with PLC control," Energy Conversion and Management, vol. 45, no. 11-12, pp. 1931-1939, 2004.

[12] M. Alata, M. A. Al-Nimr, and Y. Qaroush, "Developing a multipurpose sun tracking system using fuzzy control," Energy Conversion and Management, vol. 46, no. 7-8, pp. 1229-1245, 2005.

[13] F. S. Tymvios, C. P. Jacovides, S. C. Michaelides, and C. Scouteli, "Comparative study of Ångström's and artificial neural networks' methodologies in estimating global solar radiation," Solar Energy, vol. 78, no. 6, pp. 752-762, 2005.

[14] R. Sorichetti and O. Perpinan, "PV solar tracking systems analysis," in Proceedings of the 22nd European Photovoltaic Solar Energy Conference (EUPVSEC '07), pp. 246-252, 2007.

[15] B. Ai, H. Shen, Q. Ban, B. Ji, and X. Liao, "Calculation of the hourly and daily radiation incident on three step tracking planes," Energy Conversion and Management, vol. 44, no. 12, pp. 1999-2011, 2003.

[16] M. Meliß, Regenerative Energiequellen: Praktikum, Springer, Berlin, Germany, 1997.

[17] B. Burduhos, D. Diaconescu, I. Vişa, and A. Duţã, "Electrical response of an optimized oriented photovoltaic system," in Proceedings of the 12th International Conference on Optimization 
of Electrical and Electronic Equipment (OPTIM '10), pp. 11381145, May 2010.

[18] I. S. Hermenean and I. Visa, "Step-tracking program synthesis of an azimuth tracked concentrating photovoltaic (CPV) system," Environmental Engineering and Management Journal, vol. 10, no. 9, pp. 1225-1234, 2011. 


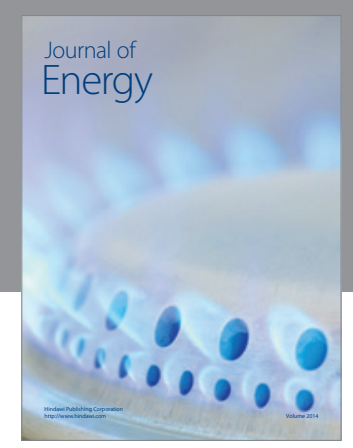

Journal of

Industrial Engineering
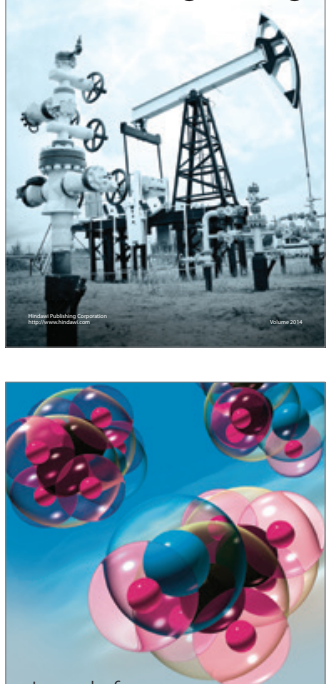

Fuels
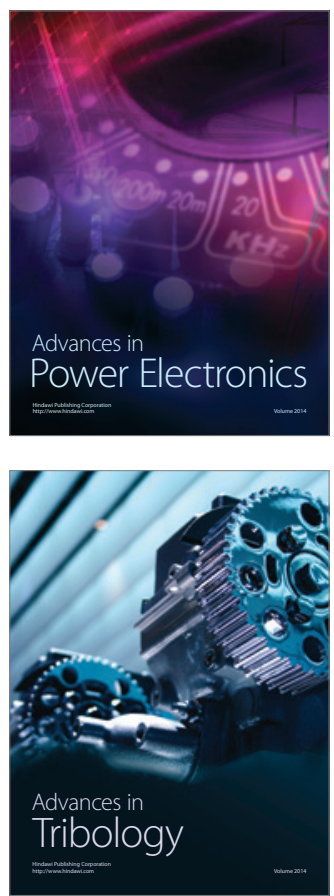

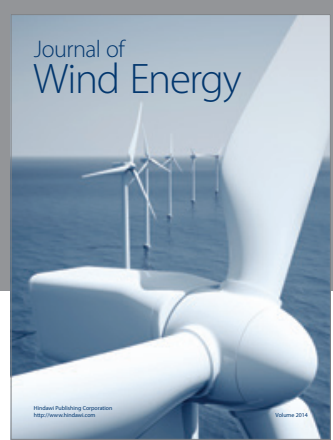

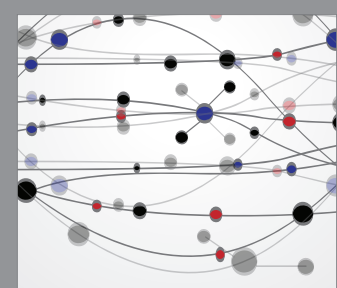

The Scientific World Journal

Submit your manuscripts at http://www.hindawi.com

Journal of

Structures
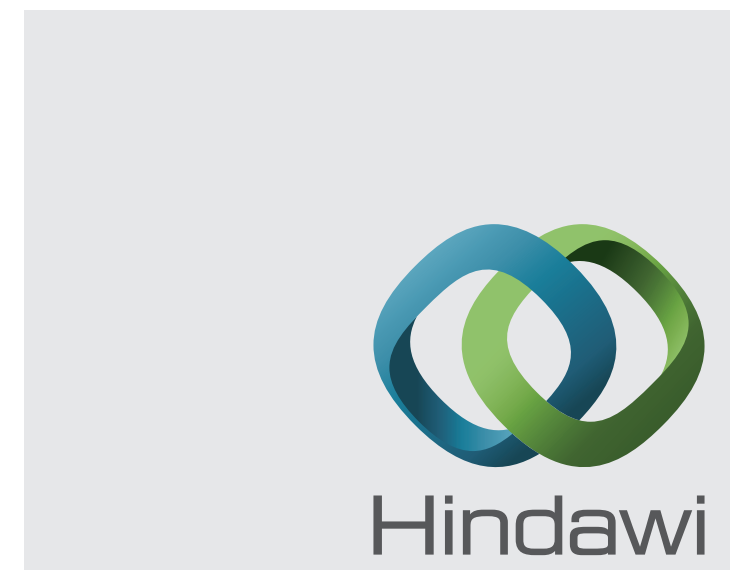

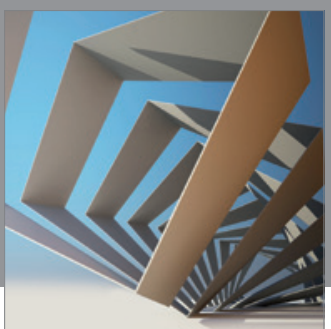

Rotating

Machinery
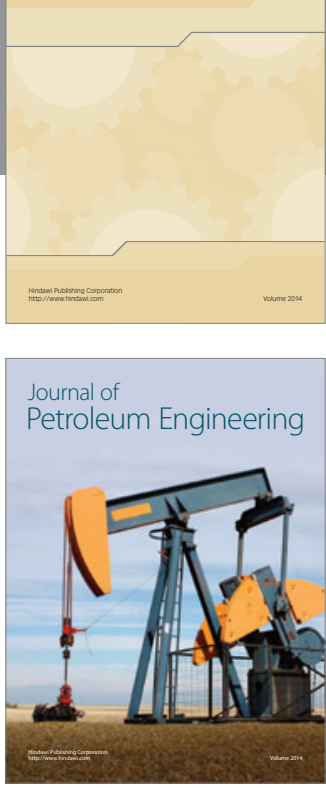

Journal of

Solar Energy
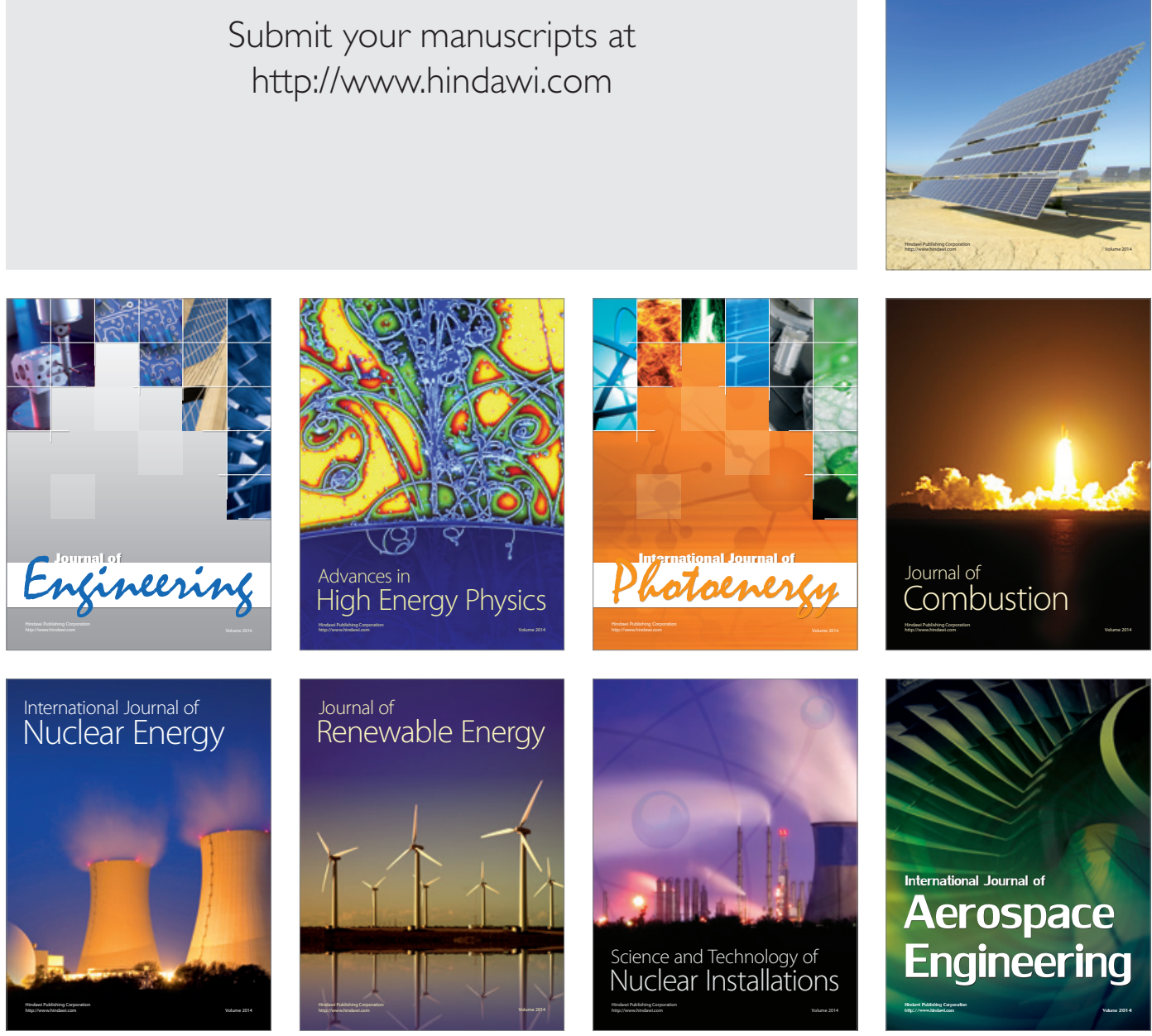